\title{
RESEARCH
}

Open Access

\section{LINC00612 enhances the proliferation and invasion ability of bladder cancer cells as ceRNA by sponging miR-590 to elevate expression of PHF14}

Liying Miao ${ }^{1+}$, Hong Yue Liu ${ }^{2,4 \dagger}$, Cuixing Zhou ${ }^{3+}$ and Xiaozhou He $\mathrm{H}^{1,3^{*}}$

\begin{abstract}
Background: Bladder cancer (BC) is a common type of cancer that involves tumors of the urinary system and poses a serious threat to human health. Long noncoding RNAs (InCRNAs) have emerged as crucial biomarkers and regulators in many cancers. Novel IncRNA biomarkers in BC urgently need to be investigated in regard to its function and regulatory mechanisms.

Methods: Identification of differentially expressed IncRNAs in BC tissue was performed via microarray analysis. To investigate the biological functions of LINC00612, loss-of-function and gain-of-function experiments were performed in vitro and in vivo. Bioinformatics analysis, dual-luciferase reporter assays, AGO2-RIP assays, RNA pull-down assays, real-time quantitative PCR (RT-qPCR) arrays, fluorescence in situ hybridization assays, and western blot assays were conducted to explore the underlying mechanisms of competitive endogenous RNAs (ceRNAs).

Results: LINC00612 was upregulated in BC tissues and cell lines. Functionally, downregulation of LINC00612 inhibited cell proliferation and invasion in vitro and in vivo, whereas overexpression of LINC00612 resulted in the opposite effects. Bioinformatics analysis and luciferase assays revealed that miR-590 was a direct target of LINC0061, which was validated by dual-luciferase reporter assays, AGO2-RIP assays, RNA pull-down assays, RT-qPCR arrays, and rescue experiments. Additionally, miR-590 was shown to directly target the PHD finger protein 14 (PHF14) gene. LNIC00612 modulated the expression of E-cadherin and vimentin by competitively sponging miR-590 to elevate the expression of PHF14, thus affecting BC cellular epithelial-mesenchymal transition (EMT).

Conclusions: Our results indicate that LINC00612 enhances the proliferation and invasion ability of BC cells by sponging miR-590 to upregulate PHF14 expression and promote BC cellular EMT, suggesting that LINC00612 may act as a potential biomarker and therapeutic target for BC.
\end{abstract}

Keywords: Bladder Cancer, LINC00612, ceRNA, EMT

\footnotetext{
* Correspondence: drxzhe@163.com

†Liying Miao, Hong Yue Liu and Cuixing Zhou contributed equally to this work.

${ }^{1}$ Department of Hemodialysis, The Third Affiliated Hospital of Soochow University, Changzhou Shi, China

${ }^{3}$ Department of Urology, The Third Affiliated Hospital of Soochow University,

Changzhou, Jiangsu Province, China

Full list of author information is available at the end of the article
}

(c) The Author(s). 2019 Open Access This article is distributed under the terms of the Creative Commons Attribution 4.0 International License (http://creativecommons.org/licenses/by/4.0/), which permits unrestricted use, distribution, and reproduction in any medium, provided you give appropriate credit to the original author(s) and the source, provide a link to the Creative Commons license, and indicate if changes were made. The Creative Commons Public Domain Dedication waiver (http://creativecommons.org/publicdomain/zero/1.0/) applies to the data made available in this article, unless otherwise stated. 


\section{Introduction}

Bladder cancer is a common type of cancer involving tumors of the urinary system, has the highest published incidence involving malignant urinary system tumors, and poses a serious threat to human health. According to data released by the US Department of Health, an estimated 76,960 patients were diagnosed with bladder cancer and 16,390 died of complications in 2016 [1]. The most common histopathological type of bladder cancer is transitional cell carcinoma (TCC), which accounts for more than $90 \%$ of bladder cancers, followed by squamous cell carcinoma, adenocarcinoma, and undifferentiated carcinoma [2]. Bladder cancer is characterized by multifocality and relapse. Most patients have non-muscle invasive bladder cancer at initial diagnosis. After active surgery and bladder perfusion therapy, relapse, infiltration and drug resistance can occur, which can threaten human health and socio-economic development [3]. The lack of specificity and sensitivity of technologies in the early diagnosis of bladder cancer, as well as the high rates of postoperative recurrence and malignant transformation following surgery, such as bladder tumor resections, are the major problems in bladder cancer diagnosis and treatment. Therefore, identification of new, highly sensitive, specific and cost-effective bladder cancer markers is needed to improve the early diagnosis rate of bladder tumors, which will have important clinical significance for ultimately improving the prognoses of patients.

LncRNAs are non-coding RNAs that are more than 200 nucleotides in length and that affect regulatory gene expression. LncRNAs lack a complete open reading frame and do not have a protein coding function. LncRNAs were first discovered by Okazaki et al. [4]. By using mouse DNA transcripts. Up to $4-9 \%$ of the sequences in the mammalian genome sequence can produce lncRNAs. LncRNAs were originally thought to be the "dark matter" or "noise" of genomic transcription and to have no biological function [5]. Later studies demonstrated that lncRNAs are involved in many important cellular functions, such as $\mathrm{X}$ chromosome silencing, genomic imprinting, chromatin modification, and transcriptional activation or inhibition, and can promote changes in molecular function in related signaling pathways, as well as alter cell life activities [6-8]. LncRNA plays a key role in the initiation, development and metastasis of bladder cancer. At present, UCN-1, PVT-1, MALAT1, SPRY4-IT1, PANDAR, H19 and other lncRNAs closely related to bladder cancer have been identified. These lncRNAs affect important biological roles, such as proliferation, apoptosis, migration and invasion of bladder cancer, and also participate in disease progression and outcomes by regulating epigenetic modifications and key cell signaling transduction pathways. In this study, gene chip screening technology was used to discover lncRNAs related to the occurrence and development of bladder cancer and to identify their functions and regulation mechanisms to promote a new understanding of the pathogenesis of bladder cancer and to guide clinical treatment.

\section{Methods \\ Microarray profiling}

TRIzol Reagent (Invitrogen, Carlsbad, CA) was used to extract total RNA which was then purified by a RNeasy Mini Kit (Qiagen, Valencia, CA). Differentially expressed lncRNAs in $\mathrm{BC}$ and normal adjacent tissues were screened by the LncRNA microarray expression profiling based on the criteria of $\log 2$ (fold change) $>1.5$ and adjusted $P<0.01$. Manufacturer's standard protocols were strictly followed. Briefly, cDNA was synthesized, labeled and purified. IncRNA microarray chips was hybridized by Cyanine-3-CTP labeled cRNA. Then after washing, samples were analyzed on the lncRNAs microarray. The differentially expressed genes were calculated and clustered by $\mathrm{R}$ program.

\section{Tissue samples}

Resected $\mathrm{BC}$ and normal adjacent tissues were collected from The Third Affiliated Hospital of Soochow University from Jan 2013 to Jan 2016. There were 13 BC samples and 8 normal adjacent tissue samples. All tissues were directly stored in liquid nitrogen at $-80^{\circ} \mathrm{C}$. Informed consent was obtained from each participant. The use of human clinical tissues was approved by the Institutional Human Experiment and Ethics Committee of The Third Affiliated Hospital of Soochow University. The Declaration of Helsinki was strictly followed during experiments.

\section{Cell line culture}

Cell lines were purchased from American Type Culture Collection (ATCC, Manassas, VA, USA) including BC cell lines (5637, UMUC3 and T24), human bladder epithelium immortalized cells (SV-HUC-1) and human embryonic kidney cell line (HEK-293). Cells were maintained in modified RPMI-1640 medium, supplemented with 10\% fetal bovine serum (FBS) including $100 \mu \mathrm{g} / \mathrm{L}$ penicillin and $100 \mu \mathrm{g} / \mathrm{L}$ streptomycin. All cell lines were grown with $5 \%$ $\mathrm{CO}_{2}$ at $37^{\circ} \mathrm{C}$.

\section{Real-time quantitative polymerase chain reaction (RT-qPCR)} TRIzol reagent (Invitrogen, Carlsbad, CA, USA) was used to isolated total RNA from tissues and cells according to the manufacturer's instructions. Moloney Leukemia Virus Reverse Transcriptase Kit (Promega, Madison, WI, USA) was then performed to reverse transcribe total RNA $(1 \mu \mathrm{g})$ to cDNA. Target primers were amplified by SYBR Green Mix (Promega). Sequences of the primers are listed in Table 1. All primers were synthesized by Shanghai Tingzhou Biological Engineering Co., Ltd. The miR-590 level was performed using TaqMan MicroRNA Assays Kit (Applied Biosystems, Carlsbad, CA, USA) according to 
Table 1 Primer used in this study

\begin{tabular}{ll}
\hline ID & Sequences \\
\hline LINC00612 forward & 5'-GGCAGAGCCATGTGTTGGATA-3' \\
LINC00612 reverse & 5'-GTGCTCCCTAATGGCTCACA-3' \\
PHF14 forward & 5'-GCAACTTGCAAGGGAACTGG-3' \\
PHF14 reverse & 5'-AAGAGGTTCCGGGATTGCC-3' \\
GAPDH forward & 5'-TGAACGGGAAGCTCACTGG-3' \\
GAPDH reverse & 5'-TCCACCACCCTGTTGCTGTA-3' \\
U6 forward & 5'-CTCGCTTCGGCAGCACA-3' \\
U6 reverse & 5'-AACGCTTCACGAATTTGCGT-3' \\
\hline
\end{tabular}

the manufacturers' instructions. All results were calculated and expressed as $2^{-\triangle \Delta C t}$. GAPDH was used as endogenous control for LINC00612 and PHF14 and U6 for miR-590. Triplicate is required for each experiment.

\section{RNA isolation of nuclear and cytoplasmic fractions}

The Nuclear/Cytoplasmic Isolation Kit (Biovision) was applied to isolate and collect cytosolic and nuclear fractions. RNA levels of LINC00612, RNU6-1(nuclear control transcript) and GAPDH (cytoplasmic control transcript) were analyzed by RT-qPCR.

\section{In situ hybridization (ISH)}

Cells were seeded onto poly-L-lysine-treated glass slides for $24 \mathrm{~h}$ after trypsinization harvest and then fixed in methanol at $-20^{\circ} \mathrm{C}$ for $5 \mathrm{~min}$. The ISH assays were performed as previously described [9]. A locked nucleic acid probe with complementarity to a section of LINC00612 (5' - TATC GAACTTTCTAGATCGGTGCAC-3' custom LNA detection probe, Exiqon) was labeled with digoxigenin antibody (Roche, 11,093,274, 1:1000) and synthesized. The intensity and the extent of staining were evaluated by 2 pathologists who were blinded to the experiment.

\section{Fluorescence in situ hybridization (FISH)}

Five thousand six hundred thirty-seven and T24 cells were fixed in $4 \%$ PFA for $15 \mathrm{~min}$. Then, $0.5 \%$ TritonX-100 was used to permeabilize the cells for $15 \mathrm{~min}$ at $4{ }^{\circ} \mathrm{C}$. Digoxigenin (DIG)-labeled LINC00612 probe or control probe mix were performed to incubate cells for $4 \mathrm{~h}$ at $55^{\circ} \mathrm{C}$. After $2 \times$ saline-sodium citrate briefly washing for $5 \mathrm{~min}$ (5-6 times), signal was detected by Horseradish peroxidase (HRP)-conjugated anti-DIG secondary antibodies (Jackson, West Grove, PA, USA). Olympus confocal laser scanning microscope was applied for image obtaining. DAPI was used to counterstain nuclear.

\section{IHC}

IHC staining was performed as previously described [9]. Briefly, the tumor tissues were cut into 4-mm-thick sections, dewaxed in xylene and rehydrated in a graded series of alcohols. Antigen was retrieved by heating the tissue sections at $100^{\circ} \mathrm{C}$ for $30 \mathrm{~min}$ in EDTA solution $(1 \mathrm{mM}$, $\mathrm{pH}$ 9.0). Cooled tissue sections were immersed in $0.3 \%$ hydrogen peroxide solution for 15 min to block endogenous peroxidase activity, rinsed with phosphate-buffered saline (PBS) for 5 min and blocked with 3\% BSA solution at room temperature for $30 \mathrm{~min}$. Subsequently, the sections were incubated with mouse monoclonal antibody against human PHF14 (1:200) at $4{ }^{\circ} \mathrm{C}$ overnight, followed by incubation with HRP-conjugated goat anti-rabbit secondary antibody. Diaminobenzene was used as the chromogen, and hematoxylin was used as the nuclear counterstain.

\section{Lentivirus production and cell transfection}

The pLVX-IRES-Puro vector for LINC00612 overexpression and lentivirus-containing short hairpin RNA (shRNA) targeting LINC00612 (top strand: Top Strand 5'-CACCG GTAGATGACAGATTAGATACCGAAGTATCTAATCT GTCATCTACC-3'; bottom strand: 5'-AAAAGGTAG ATGACAGATTAGATACTTCGGTATCTAATCTGTCAT CTACC-3') were purchased from Genelily BioTech Co., Ltd., (Shanghai, China). The cells were selected by puromycin $(2 \mu \mathrm{g} / \mathrm{mL})$ for 2 weeks at $48 \mathrm{~h}$ after transfection. Cell lines with stable LINC00612 silence or overexpression was then constructed. RT-qPCR was performed to verify the transfection efficiency. The miR-590 mimic, miR-590 inhibitor, and negative control (NC) oligonucleotides were obtained from Tingzhou Biological Engineering Co., Ltd. (Shanghai, China). Abovementioned oligonucleotides and plasmids were transfected by using Lipofectamine 3000 (Invitrogen). The manufacturer's instructions were strictly followed.

\section{Cell counting Kit-8 (CCK8) and Colony formation assay}

Cells $\left(2 \times 10^{4}\right.$ cells $\left./ \mathrm{ml}\right)$ were seeded onto 96-well plates $(100 \mu \mathrm{L} /$ well $)$ and then placed in an incubator with $5 \%$ $\mathrm{CO}_{2}$ at $37^{\circ} \mathrm{C}$ for $24 \mathrm{~h}$. After the cells were cultured for 5 days, $10 \mu \mathrm{l}$ of CCK8 solution was added to each well. The absorbance values at a wavelength of $450 \mathrm{~nm}$ were measured to evaluate cell viability. For colony formation assay, BC cells colon spheres were generated as previously described [10]. Briefly, cells (500 cells/well) were seeded in to 6-well plates for $24 \mathrm{~h}$. Cells were incubated for 2 weeks, then fixed in methanol and stained with $0.1 \%$ crystal violet. Quantity One software (Bio-Rad, Hercules, CA, USA) was used to count colonies. Triplicate is required for each experiment.

\section{Transwell assay}

Transwell chambers $(8-\mu \mathrm{m}$ pore size; Corning Costar, Cambridge, MA, USA) was applied to measure cell invasion ability. Instruction was strictly followed. Cells were suspended in serum-free RPMI-1640 medium, then seeded into the upper chamber. Serum (20\%) was supplemented into 
A
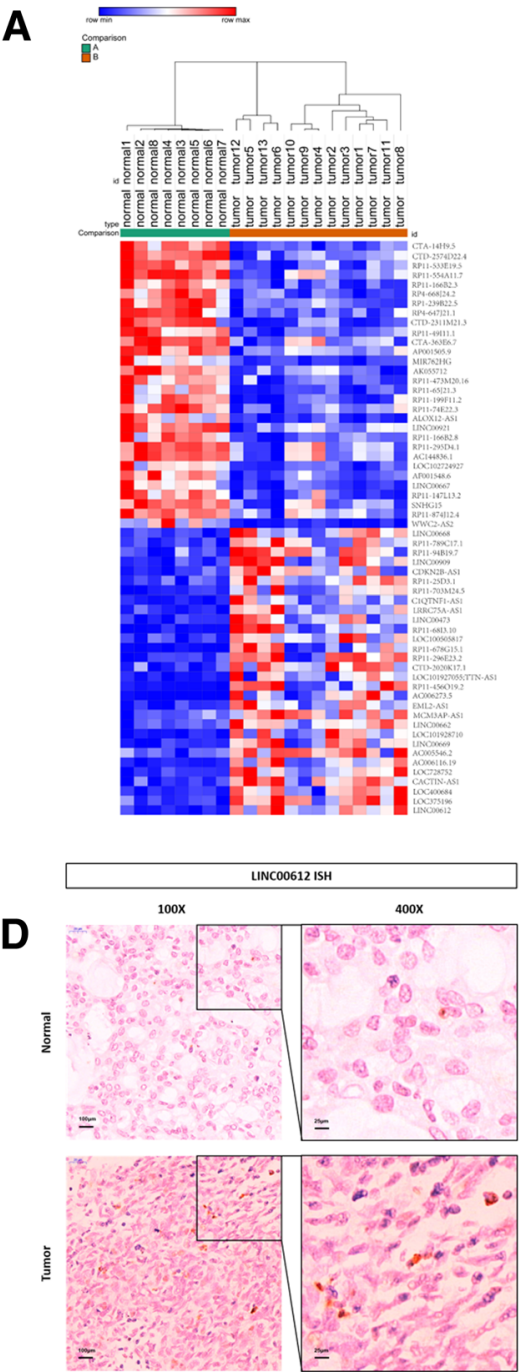

E

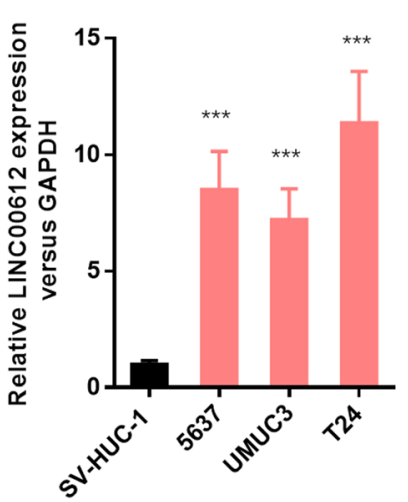

B

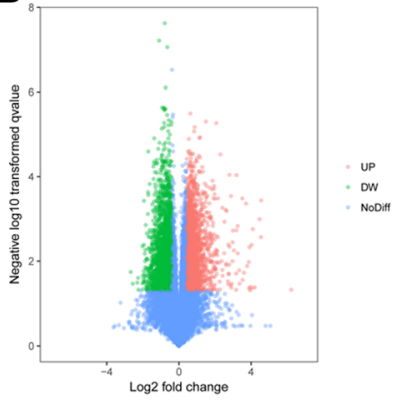

C

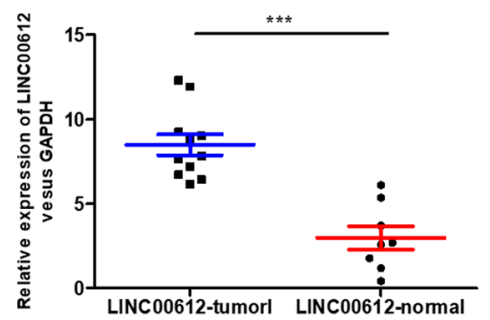

$\mathbf{F}$

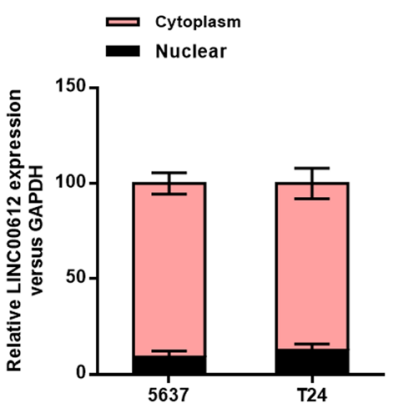

G
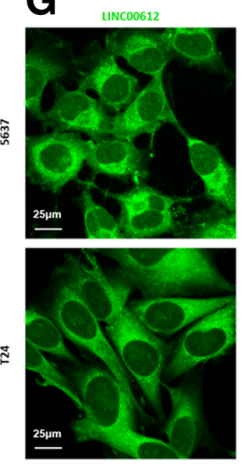
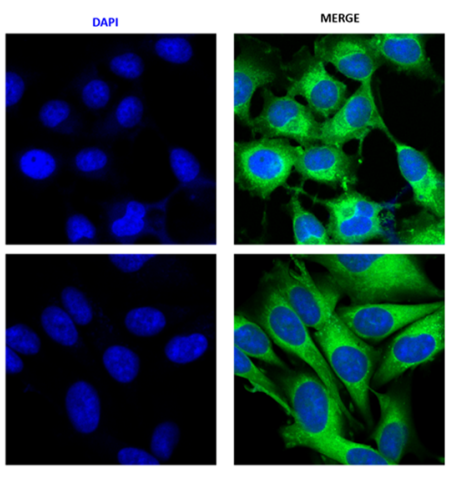

Fig. 1 LINC00612 is upregulated in bladder cancer (BC) tissues and cell lines. a Heat map with hierarchical clustering of the top 60 differentially expressed IncRNAs between BC samples and normal samples (> 1.5-fold; $P<0.05$ ). $\mathbf{b}$ LncRNAs (with fold change $>1.5$ and $P<0.05$ ) plotted as a volcano plot. c RT-qPCR was performed to validate LINC00612 expression in BC samples and normal samples ( $n=13 \mathrm{Vs} . n=8$, respectively). ${ }^{* * *} P<0.001$. d In situ hybridization detection of LINC00612 in BC tissue and normal tissue ( $\times 100$ and $\times 400)$. e RT-qPCR was performed to measure the relative expression of LINC00612 in BC cell lines (5637, UMUC3 and T24) and human bladder epithelium immortalized cells (SV-HUC-1). The majority of LINC00612 was located in the cytoplasm, according to the nuclear mass separation assay (f) and fluorescence in situ hybridization (g) 
the lower chamber that containing RPMI-1640 medium which was regarded as a chemoattractant. After $48 \mathrm{~h}$ incubation, the filters were fixed in methanol and stained with $0.1 \%$ crystal violet. The upper faces of the filters were gently abraded. Cells migrated across the lower faces of filters were imaged and counted under the microscope. Triplicate is required for each experiment.

\section{Western blot analysis}

Western blot analyses were performed according to standard protocols as previously described [11].

Anti-E-cadherin, Anti- $N$-cadherin, Anti-vimentin and Anti-PHF14 were purchased from Sigma.

\section{Luciferase reporter assays}

The reporter vector pmirGLO-LINC00612-wt was formed by cloning LINC00612 cDNA which contains predictive binding site of miR-590 into the pmirGLO Dual-Luciferase miRNA Target Expression Vector (Promega). The vector pmirGLO-LINC00612-Mut was inserted by the mutant LINC00612 that containing point mutations of the miR-590 seed region binding site. HEK-293FT cells were cultured and co-transfected with pmirGLO-LINC00612-3'-UTR vectors including wild-type or mutant fragments, miR-590 and miR-NC. Likewise, wild-type and mutant PHF14 3 '-UTR fragments were cloned into the pmirGLO vector. The miR-590 or miR-NC was co-transfected with PHF14-wt or PHF14-Mut vector into HEK-293FT cells using Lipofectamine 3000 (Invitrogen). The Dual Luciferase Reporter Assay System (Promega) was applied at $48 \mathrm{~h}$ after transfection according to the manufacturer's instructions. Triplicate is required for each experiment.

\section{RNA immunoprecipitation}

The EZMagna RIP Kit (Millipore) was applied according to the manufacturer's protocol. Complete RNA immunoprecipitation (RIP) lysis buffer was used to lyse BC cells. Magnetic beads conjugated with Anti-Argonaute 2 (AGO2) or control anti-IgG antibody were performed in incubation of cell extract. The cell extract was incubated for $6 \mathrm{~h}$ at $4{ }^{\circ} \mathrm{C}$. Then RT-qPCR analysis for purified RNA was conducted as removal of proteins of the beads had been done.

\section{RNA pull down assay}

Briefly, T24 cells were transfected with the 3'end biotinylated miR-590 or miR-590-mut or several candidate miRNAs for $24 \mathrm{~h}$ at a final concentration of $20 \mathrm{nmol} / \mathrm{L}$. Then, the cells were incubated in the cell lysate with streptavidin-coated magnetic beads (Ambion, Life Technologies). The biotin-coupled RNA complex was pulled down and analysis of the abundance of LINC00612 in bound fractions was then conducted by RT-qPCR. The pull-down assay was performed as previously described [12].

\section{Xenograft tumor model}

Xenograft tumor model was performed in BALB/c-nude mice (4-5 weeks of age) which were purchased from Shanghai SLAC Laboratory Animal Co., Ltd., China. The experimental procedures were approved by the Institutional Animal Care and Use Committee of our institution. Tumor growth was monitored every 5 days; tumor volumes were estimated by length and width. One month later, the mice were sacrificed, then tumors were excised and weighed.

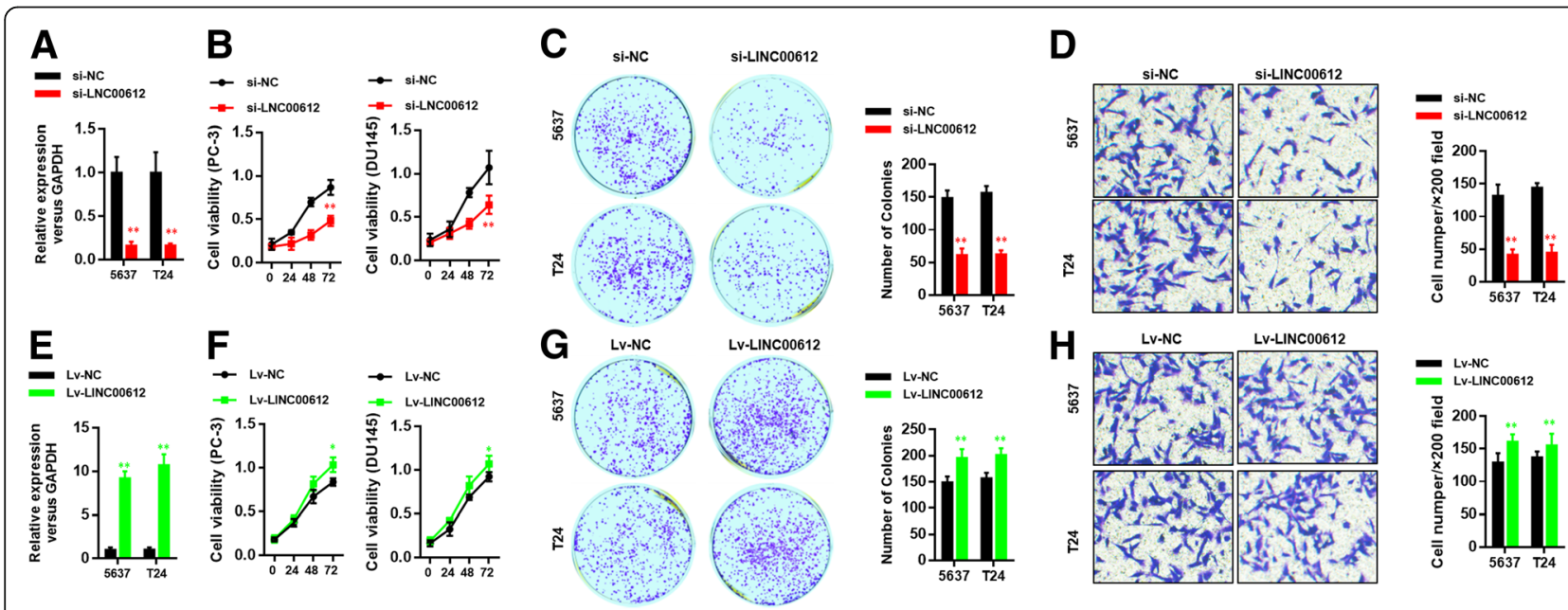

Fig. 2 LINC00612 promotes cell proliferation and invasion of BC cells in vitro. a Efficiency of LINC00612 expression in LINC00612 downregulated 5673 and T24 cells was evaluated via RT-qPCR. ${ }^{* *} P<0.01$. b Cell viability was measured via CCK8 assays. ${ }^{*} P<0.01$. c, d Representative results of colony formation and transwell assays of 5673 and T24 cells after shLINC00612 or shNC transfection. e Efficiency of LINC00612 expression in LINC00612 overexpressed 5673 and T24 cells was evaluated via RT-qPCR. ${ }^{* *} P<0.001$. f Cell viability was measured via CCK8 assays. ${ }^{*} P<0.05$. g, $\mathbf{h}$ Representative results of colony formation and transwell assays of 5673 and T24 cells after Lv-LINC00612 or LV-NC transfection. N=3 independent experiments 
Abdominal metastasis model

After anesthetization, a left lateral flank incision was operated on the mice. The spleen was then exteriorized. About $100 \mu \mathrm{l}$ of Hank's balanced salt solution that containing T24-Luc-vector and T24-Luc-siLINC00612 cells $(8 \times 10$ [6]) were injected into the spleen parenchyma by 25 -gauge needle. The IVIS bioluminescence imaging system (Caliper Life Sciences) was performed to collect bioluminescence images after day 28. All experiments were approved by the relevant guidelines of The Third Affiliated Hospital of Soochow University.

\section{Statistical analysis}

SPSS 22.0 statistical software package and GraphPad Prism 7.0 were applied for statistical analyses.

All data are represented as mean \pm standard deviation (SD). To compare two or more groups, the Student's t-test or one-way analysis of variance (ANOVA) were performed for differences analysis. Differences were considered statistically significant when $P<0.05$.

\section{Results}

LINC00612 was up-regulated in BC tissues and cell lines

A total of 60 lncRNAs (fold change $>1.5$, Padj $<0.01$ ) differentially expressed in BC tissues were screened (Fig. 1a \& b). As shown in the heat map, LINC00612 was significantly up-regulated in tumor tissues compared with that in normal tissues (Fig. 1a). The results of ISH and RT-RT-qPCR revealed that the expression of LINC00612 was higher in tumor tissues than that in normal tissues (Fig. 1c \& d). According to the results of RT-qPCR, the expression of LINC00612 in BC cell lines (5637, UMUC3 and T24) was significantly elevated compared with that in human bladder epithelium immortalized cells (SV-HUC-1) (Fig. 1e), and there was a statistically significant difference $(P<0.05)$. The subcellular localization of LINC00612 in BC

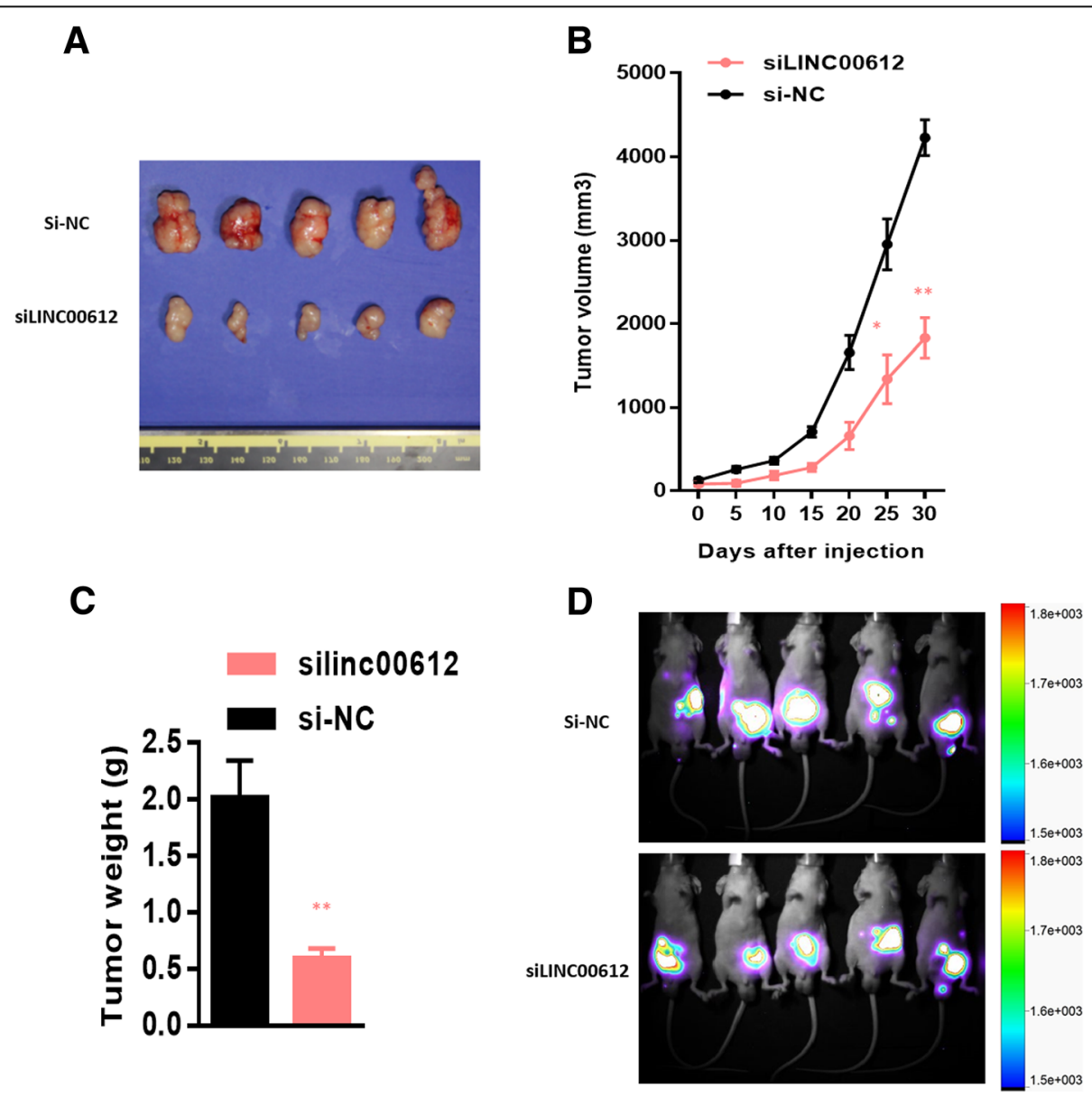

Fig. 3 Ablation of LINC00612 inhibits proliferation and metastasis in vivo. a Nude mice were subcutaneously injected with T24 cells transfected with the shLINC00612 vector and control vector. Tumors were removed after 1 month. Both tumor volume (b) and tumor weight (c) were analyzed between the shLINC00612 and control groups. d Bioluminescent imaging of shLINC00612 and luciferase expressing control T24 cells transplanted in nude mice. ${ }^{* *} P<0.01$ 
cell lines was determined using the nuclear mass separation assay and FISH, and it was found that LINC00612 was mainly located in the cytoplasm (Fig. If \& g).

\section{Ablation of LINC00612 inhibited cell viability and invasion of $B C$ cells}

Loss-of-function experiments was used to determine whether LINC00612 influences BC cell proliferation and invasion. RT-qPCR was applied to verify knockdown efficiency of LINC00612. After transfection with the vector containing shLINC00612, the expression of LINC00612 in 5637 and T24 cells was obviously reduced compared with that in empty vector group (Fig. 2a). To evaluate cell viability, we subsequently performed CCK8 and colony formation assays. The results showed that cell growth and colony formation were strongly inhibited by ablation of LINC00612 (Fig. 2b \& c). Also, knockdown of LINC00612 weakened the cell invasive and migratory capacity (Fig. 2d). Consistently, when we transfected the vector that containing LINC00612 overexpression plasmid into BC cells, the expression of LINC00612 in 5637 and T24 cells was significantly increased compared with that in empty vector group (Fig. 2e). Cell growth and colony formation were thus enhanced (Fig. $2 f$ \& g) as well as the cell invasive and migratory capacity (Fig. 2h). The differences were statistically significant $(P<0.05)$.

\section{In vivo verification of influence of LINC00612 on cell proliferation and invasion ability}

To evaluate the influence of LINC00612 on proliferation and invasion ability, BALB/c-nude mice were injected subcutaneously with the T24 cells with stable downregulation of LINCOO612 and corresponding control cells. As expected, the transplanted tumor with the down-regulation of LINC00612 had a smaller volume and lower weight than that in control group (Fig. 3a, b \& c). To investigate the effect of LINCOO612 on metastases, an in vivo abdominal metastasis model was performed. We injected approximately $1 \times 10^{7}$ LINC00612 down-regulated and control T24 cells into spleen of BALB/c-nude mice and used bioluminescence imaging to evaluate abdominal metastasis. One month after injection, bioluminescence imaging confirmed that LINCO0612 ablation significantly

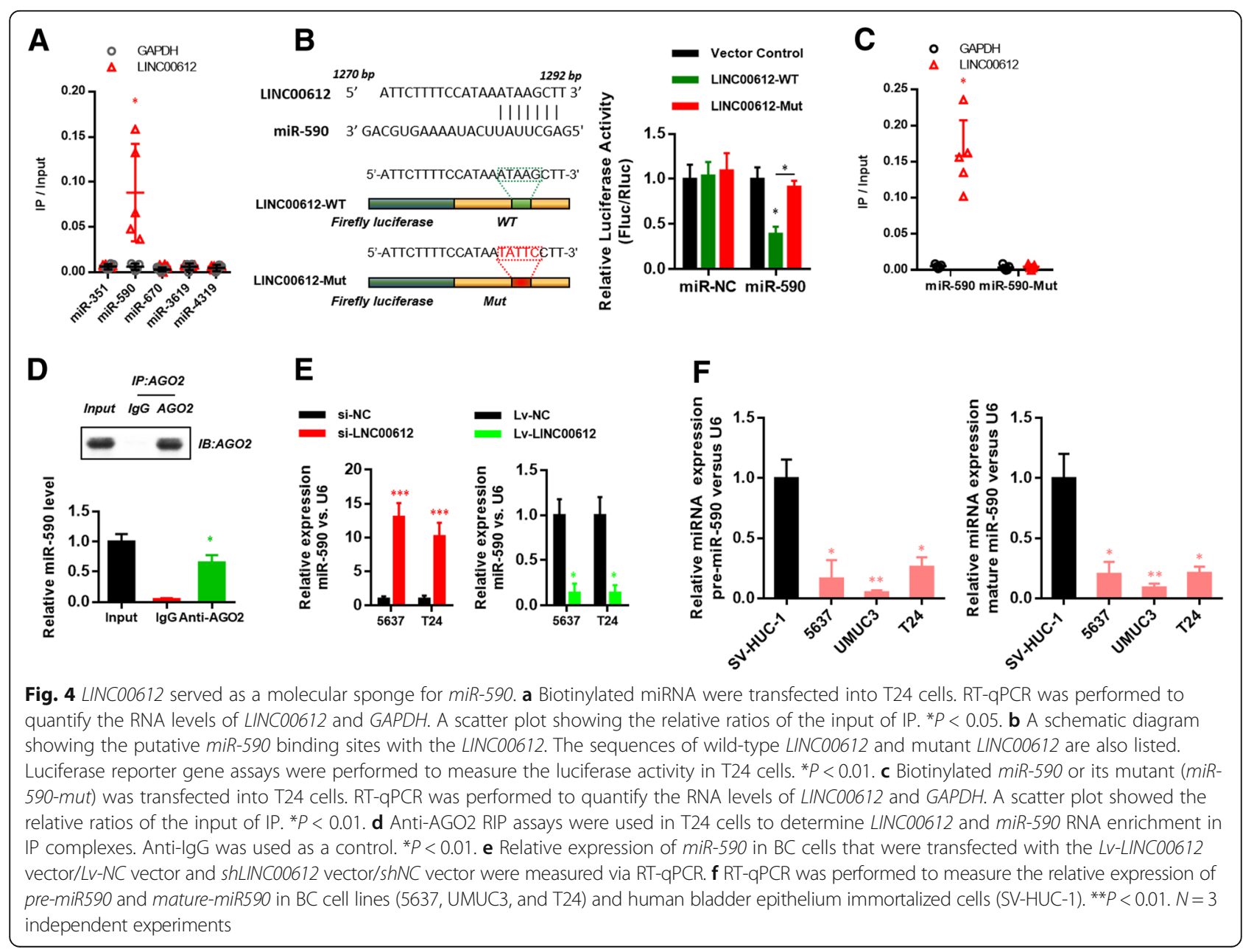


inhibited metastasis (Fig. 3d). The differences were statistically significant $(P<0.05)$.

\section{LINC00612 played a role in BC as an endogenous competitive RNA of miR-590 and regulated target gene PHF14 of miR-590}

The competitive endogenous RNA (ceRNA) is a well-known regulatory mechanism of lncRNA. LncRNA sponges a variety of miRNAs to inhibit its expression and reduce the regulatory effect on target mRNA. The target recognition sequence of LINC00612 miRNA was analyzed via bioinformatics (RegRNA $2.0 \mathrm{http}$ ://regrna2. mbc.nctu.edu.tw/detection.html; Lncrnadb http://www. lncrnadb.org; miRcode http://www.mircode.org/), and it was found that several miRNAss had a complementary sequence to LINC00612. RNA pull-down was performed to screening the most relevant target miRNA. The result indicated that LINC00612 was more enriched in the miR-590 compared with other miRNAs (Fig. 4a). To prove the finding, LINC00612 cDNA was cloned into the luciferase gene (pGL3-PVT1-214-wt) and co-transfected with $m i R-590$ or miR-NC. The results revealed that the luciferase activity in miR-590 group was significantly reduced compared with that in miR-NC group. At the same time, the miR-590 binding site was mutated, and the pGL3-PVT1-214-mut vector was produced. The results showed that the vector after mutation had no significant influence on the luciferase activity in miR-590 group (Fig. 4b). The results of RNA pull-down assay manifested that LINC00612 was more enriched in the wild-type miR-590 compared with that in the mutant-type miR-590 with broken LINC00612 binding site (Fig. 4c). RNA induced silencing complexes (RISCs) is formed by miRNA ribonucleoprotein complexes (miRNPs) which is present in anti-Ago2 immunoprecipitates. Therefore, antiAgo2 immunoprecipitates contain miRNAs and their interacting RNA-components [13-15]. RIP assay was performed using anti-AGO2 in the T24 extract, and it was found that LINC00612 and miR-590 were enriched preferentially in miRNPs containing AGO2 compared with anti-IgG immunoprecipitates (Fig. 4d). The RT-qPCR results showed that down-regulation of LINC00612 could cause the increase in miR-590 expression, while overexpression of LINC00612 in $\mathrm{BC}$ cells could lead to the down-regulation of miR-590

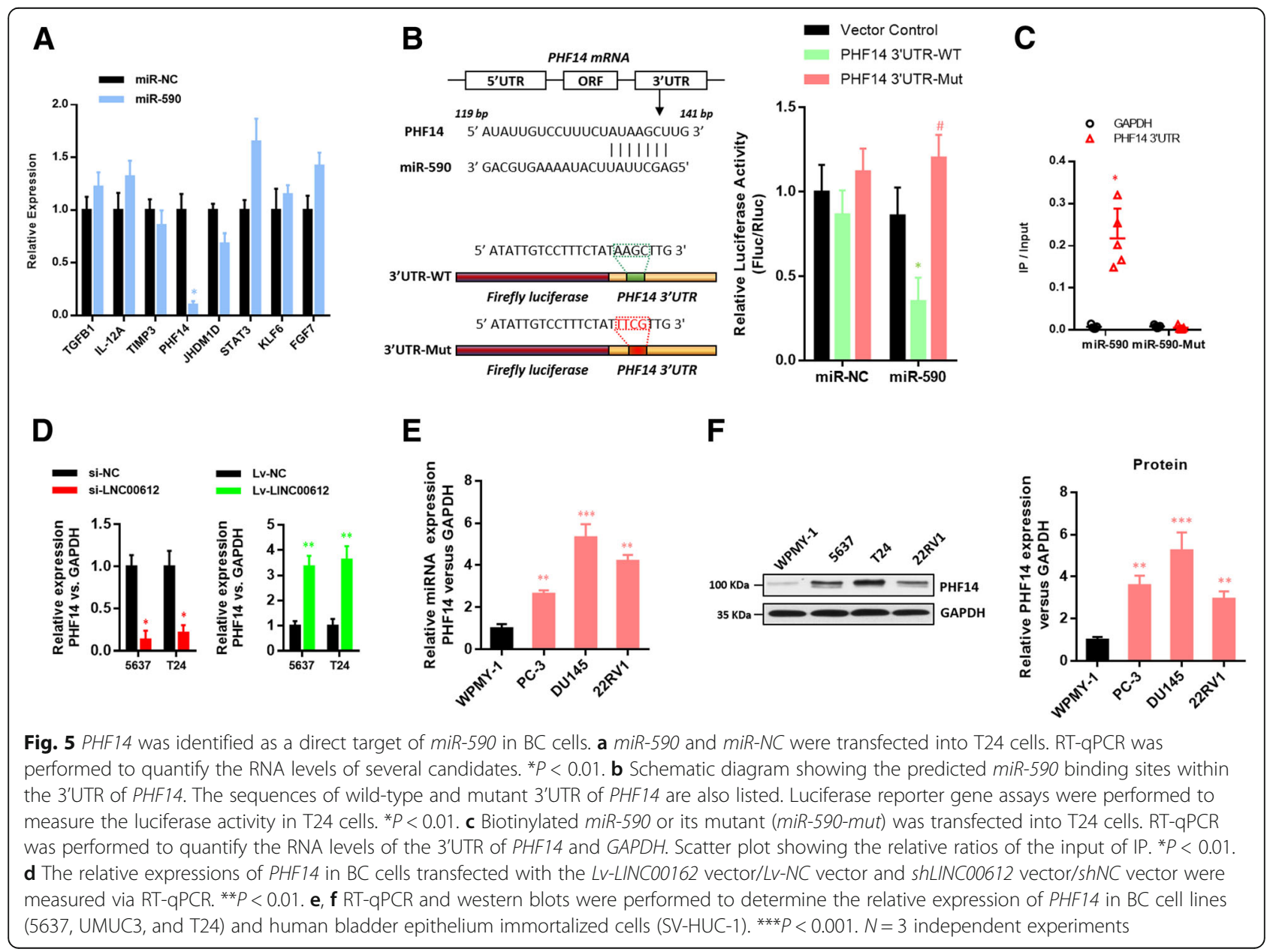


expression (Fig. 4e). RT-qPCR results displayed that the expression of pre-miR-590 and mature miR-590 in BC cell lines (5637, UMUC3 and T24) was significantly reduced compared with that in human bladder epithelium immortalized cells (SV-HUC-1) (Fig. 4f).

According to the prediction via bioinformatics (Targetscan 7.2 http://www.targetscan.org/vert_72/; miRDB http://www.mirdb.org/; miRTarBase http://mirtarbase. mbc.nctu.edu.tw/php/index.php), there were binding sites in several genes' 3'-UTR with miR-590. Then RT-qPCR was used to screening. Finally, PHF14 was identified as target gene (Fig. 5a). Then the luciferase assay confirmed that the $3^{\prime}$-UTR of wild-type PHF14 could significantly lower the luciferase activity in $m i R-590$ group without significant influence on the luciferase activity in miR-NC group. The $3^{\prime}$-UTR of mutant-type PHF14 had no obvious influence on the luciferase activity in miR-590 group (Fig. 5b). RNA pull-down assay confirmed that PHF14 3'-UTR was more enriched in the wild-type miR-590 compared with that in the mutant-type miR-590 with broken PHF14 3'-UTR binding site (Fig. 5c). Whether PHF14 could be regulated by the expression of LINC00612 was verified then. According to the RT-qPCR results, the overexpression or down-regulation of LINC00612 could increase or decrease the mRNA expression in PHF14 (Fig. 5d).
RT-qPCR and western blotting results verified that the expression of PHF14 in BC cell lines (5637, UMUC3 and T24) was significantly increased compared with that in human bladder epithelium immortalized cells (SV-HUC-1) (Fig. 5e \& f). Moreover, the down-regulation of LINC00612 expression in $\mathrm{BC}$ cells could reverse the promotion on cell viability (Fig. 6a \& c) and invasive and migratory capacity (Fig. 6e) caused by PHF14 overexpression. Accordingly, the overexpression of LINC00612 could reverse inhibition on cell viability (Fig. $6 \mathrm{~b} \& \mathrm{~d}$ ) and invasive and migratory capacity caused by PHF14 knock-down (Fig. 6f).

\section{LINC00612/miR-590/PHF14 axis regulated BC cell epithelial-mesenchymal transition (EMT)}

EMT is one of major cause that epithelial-derived malignant cells gained increased migration and invasion ability. To evaluate the effect on EMT of LINC00612, western blotting assay was performed. It could be seen in the result that knock-down of LINC00612 enhanced the expression of the epithelial marker E-cadherin and weakened the expression of $\mathrm{N}$-cadherin and the mesenchymal marker vimentin (Fig. 7a). Correspondingly, overexpression of LINC00612 inhibited the expression of E-cadherin and promoted the expression of $\mathrm{N}$-cadherin and vimentin (Fig. 7b). This conclusion also had been manifested by FISH (Fig. 7c). We further explored the

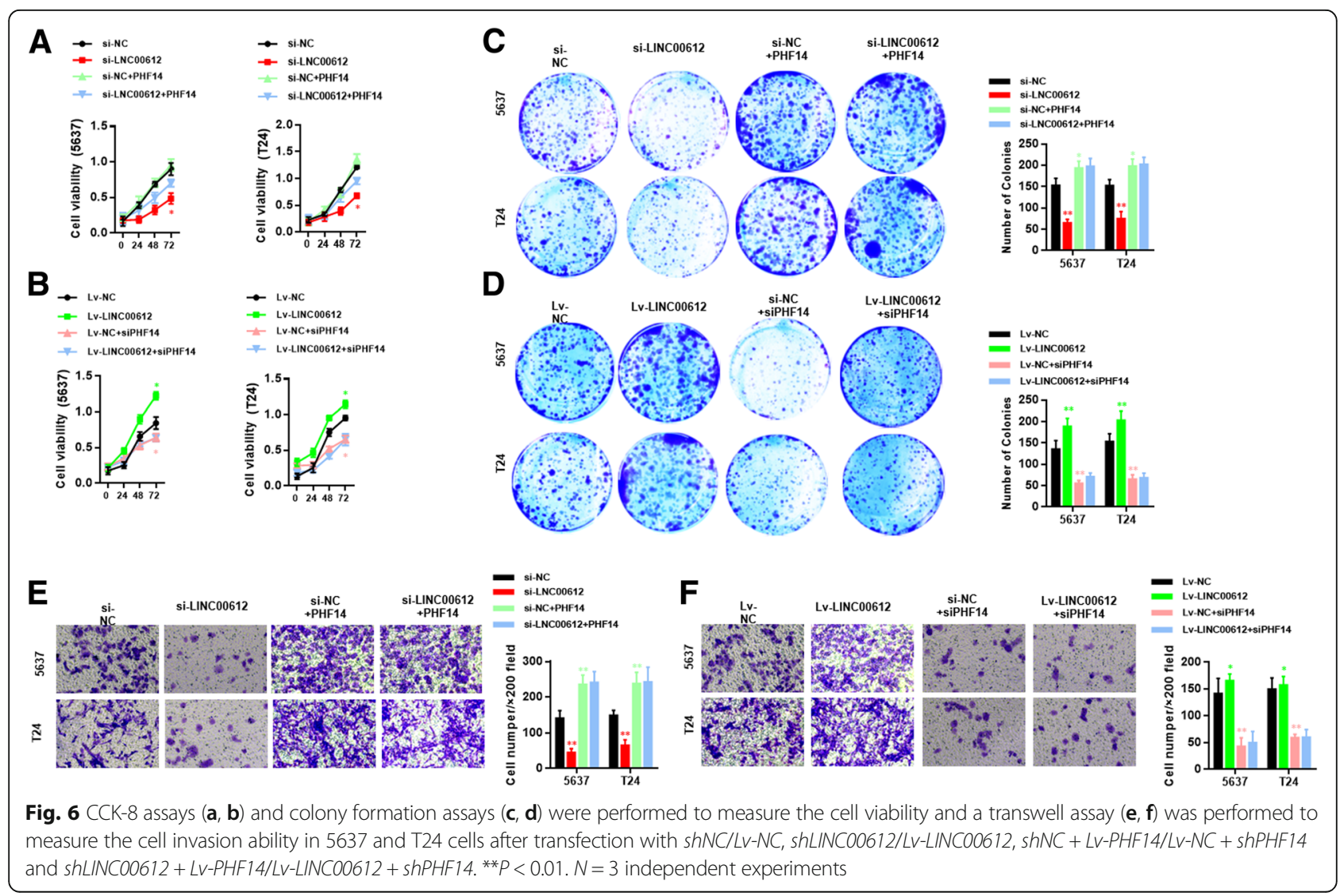




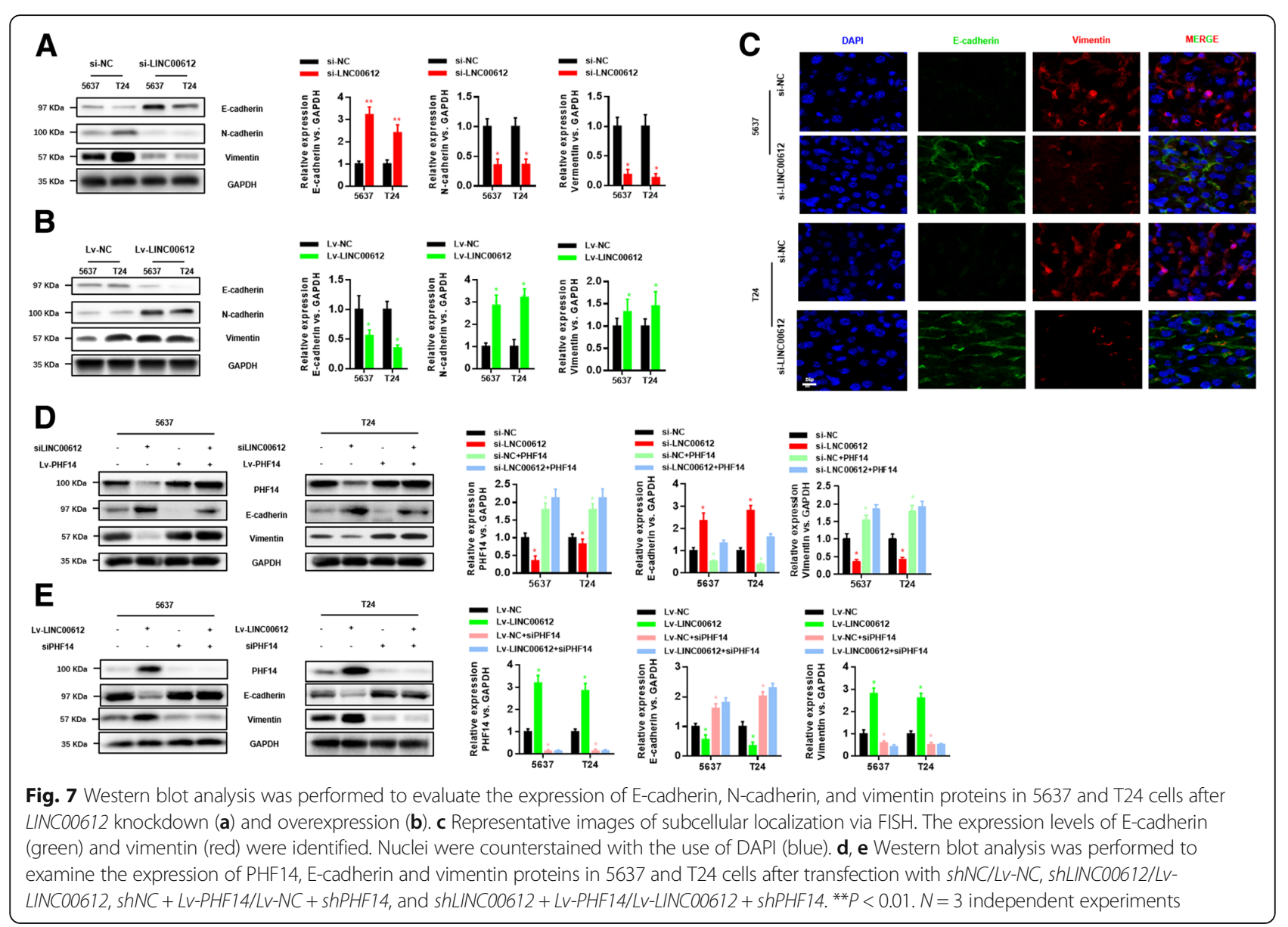

role of LINC00612/miR-590/PHF14 pathway that affect EMT. The ablation of LINCO0612 could reverse the promotion on expression of vimentin and inhibition on expression of E-cadherin caused by PHF14 overexpression (Fig. 7d). The similar results were also observed in Lv-LINC00612 + siPHF14 group (Fig. 7e). The results suggested that LINC00612/miR-590/PHF14 axis is a novel regulator in $\mathrm{BC}$ progression and metastasis.

\section{In vivo verification of impact of LINC00612/miR-590/ PHF14 axis}

In nude mice xenografts, the tumor volume and mass could be significantly affected by modulation of miR-590 and PHF14 in T24 which subcutaneously injected to nude mice $(P<0.05)$. The transplanted tumor with the transfection of siLINC00612 had a smaller volume and lower weight than that in control group. However, the tumor suppression effect in vivo caused by siLINC00612 was neglected when co-transfected with siLINCO0612+ miR-590 inhibitor or siLINC00612+Lv-PHF14. The differences on tumor volume and mass between co-transfecting group and their negative control group were not statistically significant. The IHC results showed that PHF14 was less expressed in siLINC00612 tumor tissue than those in control, $L v$-PHF14 and siLINC00612 + Lv-PHF14 tumor tissues. (Fig. 8). All these results suggested that LINC00612/miR-590/ PHF14 axis could eventually modulate the proliferation in $\mathrm{BC}$ cells.

\section{Discussion}

In recent years, several lncRNAs related to the development of bladder cancer have been identified. Wang et al. [16] reported that exogenous expression of urothelial cancer associated 1 (UCA1) in the bladder transitional cell carcinoma cell line BLS-211 enhanced the proliferation, migration, invasion and drug resistance of bladder cancer cells. Wu et al. [17] reported that $U C A 1$ can regulate the proliferation of bladder cancer cell lines through the PI3KAKT-mTOR signaling pathway. Furthermore, they also confirmed that the transcription factor CCAAT/ enhancer-binding protein (C/EBP)- $\alpha$ can affect the expression level of UCA1. UCA1 inhibits the growth of bladder cancer cells by inhibiting the expression of the transcription factor C/EBP- $\alpha$. In addition, a number of studies have shown that $U C A 1$ expression can be detected in tumors of bladder cancer patients, as well as in their urine, and exhibits tumor tissue specificity [18-20]. UCA1 exhibits 


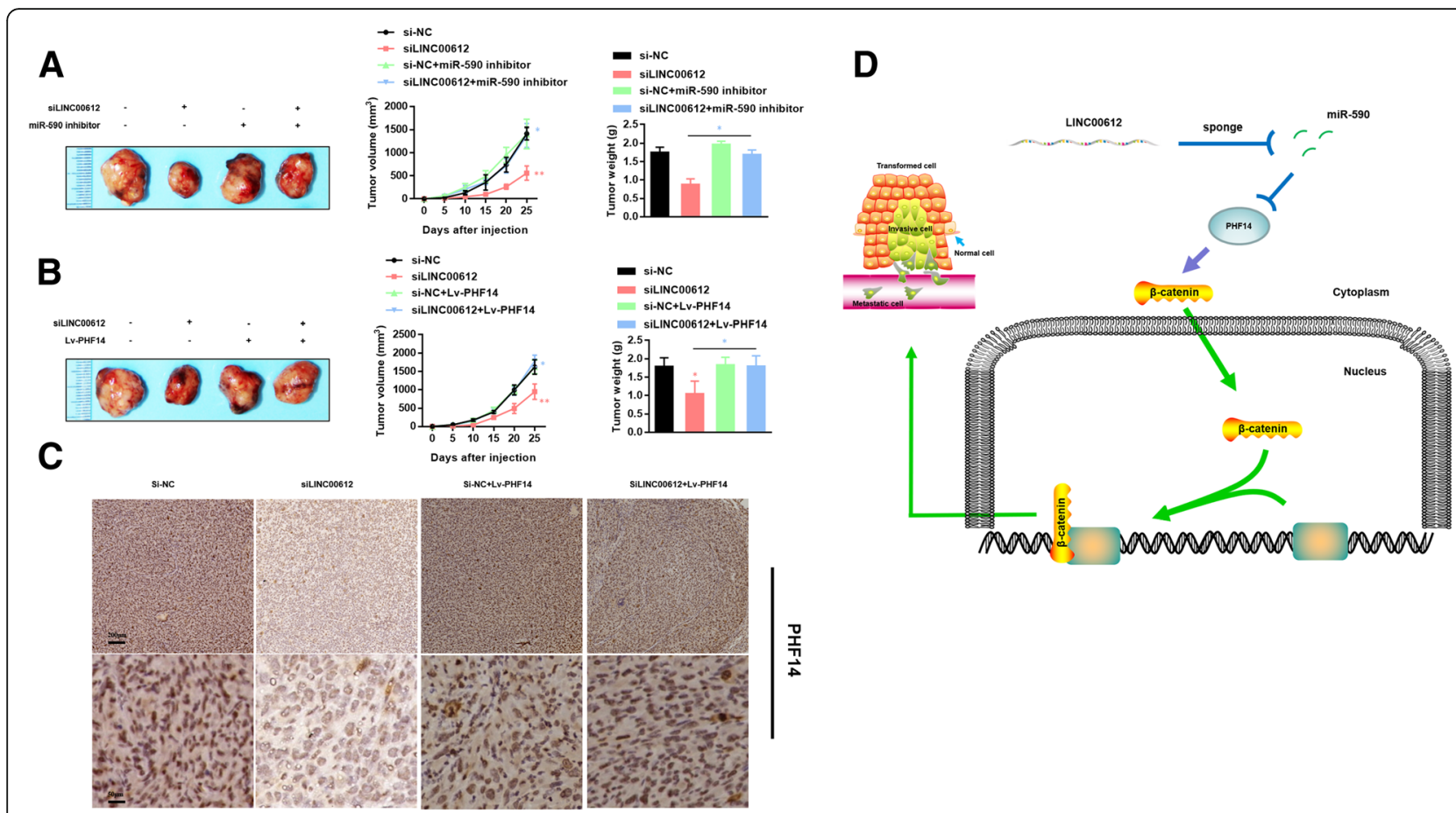

Fig. 8 Tumors were collected from nude mice injected with T24 cells transfected with siNC, siLINC00612, miR-590 inhibitor + siNC, miR-590 inhibitor + siLINC00612 (a), and siNC, siLINC00612, Lv-PHF14 + siNC, Lv-PHF14 + siLINC00612 (b). Tumor volume was analyzed every 5 days. Tumor weight was measured 30 days after the tumor transplantation. The differences in tumor volume and mass between the co-transfecting group (siLINC00612 + miR-590 inhibitor and siLINC00612 + LV-PHF14) and the negative control group were not statistically significant. ${ }^{*} P<0.05$, compared with the NC group. $\mathbf{c}$ IHC detection of PHF14 in paraffin-embedded tissue sections. d Possible molecular mechanisms of the LINC00612/miR-590/ PHF14 axis in bladder cancer: LINC00612 competitively binds to miR-590, which could directly combine with PHF14. PHF14 could enhance the proliferation and invasion ability of bladder cancer cells by activating the WNT pathway via the promotion of migration of $\beta$-catenin from the cytoplasm into the nucleus. LINC00612 weakens the inhibiting effect of miR-590 on PHF14

high sensitivity, strong specificity, and stable experimental results with respect to bladder cancer. Chen et al. [21] used microarray analysis to screen differentially expressed lncRNAs and demonstrated that the expression level of the lncRNA $n 336928$ in bladder cancer tissues was significantly higher than that in paracancerous tissue and that expression of the lncRNA $n 336928$ was positively correlated not only with the stage and grade of bladder tumors but also with age, gender, smoking status, tumor size, and tumor number. Survival analyses revealed that the 5-year survival rate of patients in the high lncRNA n336928 expression group was significantly lower than that in the low expression group. Liu et al. [22] reported that expression of SPRY4-IT1 in bladder cancer tissues and bladder cancer cell lines was higher than that in paracancerous normal tissues and normal bladder epithelial cells. Downregulation of SPRY4-IT1 expression by siRNA interference significantly inhibited the proliferation and migration of bladder cancer cells and promoted apoptosis of bladder cancer cells [23]. To date, little is known about the role of LINC00612 in tumors. In this study, we found that LINC00612 is significantly upregulated in bladder cancer tissues and cell lines. Further studies confirmed that
LINC00612 could promote tumor proliferation and invasion in vivo and in vitro, suggesting that LINC00612 may be a potential target for observation and treatment of bladder cancer.

Researchers at Harvard University proposed the ceRNA hypothesis in 2011, arguing that there is a pattern of interactions between miRNA and mRNA. The hypothesis further states that various types of RNA molecules (including mRNA, lncRNA, etc.) can regulate each other by competitively inhibiting miRNAs via common microRNA response elements (MRE) [24]. The stability and transcription of cytoplasmic lncRNA can be modified by trapping miRNAs, thereby altering signaling pathways. In this study, we found that LINC00612 was primarily localized in the membrane, which indicated that the ceRNA mechanism may exist. Subsequently, bioinformatic predictions, luciferase reporter gene experiments, RIP experiments, and RNA pull-down experiments revealed that LINC00612 directly binds and sponges miR-590. miR-590 has been shown to play an important role in a variety of cancers, and its function has been described as being carcinostatic in breast cancer, osteosarcoma and lung cancer [25-28]. However, some studies 
reached different conclusions. These studies suggested that miR-590 could promote tumor cell proliferation and enhance tolerance to radiotherapy [6-8]. The effect of miR-590 in bladder cancer remains unclear. In this study, we showed that miR-590 could be sponged by LINC00612 and therefore counter the carcinogenic effect of LINC00612, which provides a basis for confirming the carcinostatic effect of $m i R-590$ in bladder cancer. Agostino [29] suggested that miR-590 can be specifically adsorbed by the IncRNA MIR205HG in head and neck squamous cell carcinomas, thereby leading to uncontrolled tumor cell proliferation. This ceRNA regulation mechanism was similar to our findings.

EMT is a biological process in which epithelial-derived malignant cells transform into mesenchymal cells with increased migration and invasion ability [30]. The characteristic changes in EMT include the loss of polarity of epithelial cells, degradation of intercellular junctions, changes in cell morphology due to the reorganization of cytoskeletal structures, and downregulation of epithelial gene expression accompanied by upregulation of mesenchymal gene expression. These changes provide the cell with a greater ability to migrate, invade and degrade the extracellular matrix [31]. During EMT, cells lose epithelial marker factors, such as E-cadherin, while mesenchymal markers increase, such as vimentin, N-cadherin, and fibronectin, and related transcription factors, including Twist, Snail, and Zeb families, are activated. The results of this study conclude that LINC00612 promotes EMT in BC cells by inhibiting the expression of the epithelial marker E-cadherin and by enhancing the expression of the mesenchymal marker vimentin, thus increasing the proliferation and invasion of $\mathrm{BC}$ cells. This regulation was competitively adjusted by miR-590, according to the ceRNA mechanism. $m i R-590$ has been previously reported as being an EMT inhibitory miRNA [32], which is in accordance with our findings. Furthermore, we demonstrated that miR-590 could directly bind to downstream PHF14 at the 3'UTR. PHF14 is involved in several signaling pathway, including the classical TGF- $\beta$ signaling pathway [33]. Several researchers have demonstrated that PHF14 is overexpressed in biliary tract cancer and lung cancer and may also be involved in tumorigenesis $[34,35]$. To measure whether the regulation of the LINC00612/miR-590/PHF14 axis modulated cellular EMT and thus modulated the proliferation and invasion of $\mathrm{BC}$ cells, rescue experiments in vitro and in vivo were conducted. When LINC00612/miR-590 and LINC00612/ shPHF14 (shLINC00612/PHF14) were cotransfected into $\mathrm{BC}$ cells, the alterations in cellular EMT, cell proliferation and invasion were restored. These results confirmed that the LINC00612/miR-590/PHF14 axis had a substantial effect on BC cellular EMT and might be a crucial modulator of cell proliferation and invasion in BC cells. At present, the function and regulation of LINC00612 in other tumors remains unclear. Meanwhile, the regulation of the LINC00612/miR-590/PHF14 axis in bladder cancer requires confirmation in large-scale clinical research studies, which will be the main aim of a future study.

\section{Conclusion}

In summary, our results suggest that the expression of LINC00612 is elevated in bladder cancer and can promote tumor cell proliferation and invasion in bladder cancer, as confirmed by in vivo and in vitro experiments. Furthermore, we suggest a possible regulatory mechanism through the sponging of miR-590 by LINC00612, leading to upregulation of PHF14, promoting tumor cellular EMT and enhancing the proliferation and invasion of bladder cancer cells. LINCO0612 may be a novel bladder cancer marker and a potential therapeutic target.

\section{Abbreviations}

BC: Bladder Cancer; ceRNAs: competitive endogenous RNAs; EMT: epithelialmesenchymal transition; FISH: Fluorescence in situ hybridization; ISH: In situ hybridization; PHF14: PHD finger protein 14; RT-qPCR: Real-Time quantitative PCR arrays

\section{Acknowledgements}

Dr. Zhuojun Zheng collaborated in the collection and interpretation of the data and contributed to the manuscript

\section{Funding}

This work was supported by the Science and Technology Bureau foundation application project of Changzhou (CJ20179028).

\section{Availability of data and materials}

The datasets used and analyzed during the current study are available from the corresponding author on reasonable request.

\section{Authors' contributions}

HY Liu and CX Zhou performed experiments, LY Miao analyzed data and wrote the paper; XZ He initiated the study, designed experiments and edit the paper. All authors read and approved the final manuscript.

\section{Ethics approval and consent to participate}

Written informed consent for the biological studies was obtained from each patient involved in the study, and the study was approved by the Ethics

Committee of The Third Affiliated Hospital of Soochow University. All animal studies were approved by the Animal Experimental Committee of The Third Affiliated Hospital of Soochow University.

\section{Consent for publication}

Written consent for publication was obtained from all the patients involved in our study. No identifiable individual patient or health person was involved in this study.

\section{Competing interests}

The authors declare that they have no competing interests.

\section{Publisher's Note}

Springer Nature remains neutral with regard to jurisdictional claims in published maps and institutional affiliations.

\section{Author details}

${ }^{1}$ Department of Hemodialysis, The Third Affiliated Hospital of Soochow University, Changzhou Shi, China. ²Department of Pharmacy, Fudan University Shanghai Cancer Center, Shanghai, China. ${ }^{3}$ Department of Urology, The Third Affiliated Hospital of Soochow University, Changzhou, Jiangsu Province, China. ${ }^{4}$ Department of Oncology, Shanghai Medical College, Fudan University, Shanghai, China. 
Received: 14 November 2018 Accepted: 21 March 2019 Published online: 02 April 2019

\section{References}

1. Smith RA, et al. Cancer screening in the United States, 2016: a review of current American Cancer Society guidelines and current issues in cancer screening. CA Cancer J Clin. 2016;66:96-114. https://doi.org/10.3322/caac. 21336.

2. Kamat AM, et al. Bladder cancer. Lancet. 2016;388:2796-810. https://doi.org/ 10.1016/S0140-6736(16)30512-8.

3. Eapen $\sqcup$, MacRae RM. Management considerations after cystectomy for bladder Cancer. Journal of clinical oncology : official journal of the American Society of Clinical Oncology. 2016;34:3228. https://doi.org/10. 1200/JCO.2016.67.9423

4. Okazaki $Y$, et al. Analysis of the mouse transcriptome based on functional annotation of 60,770 full-length cDNAs. Nature. 2002;420:563-73. https://doi. org/10.1038/nature01266.

5. Kotake $Y$, et al. Long non-coding RNA ANRIL is required for the PRC2 recruitment to and silencing of p15(INK4B) tumor suppressor gene. Oncogene. 2011;30:1956-62. https://doi.org/10.1038/onc.2010.568.

6. Castro-Oropeza R, Melendez-Zajgla J, Maldonado V, Vazquez-Santillan K. The emerging role of IncRNAs in the regulation of cancer stem cells. Cell Oncol. 2018. https://doi.org/10.1007/s13402-018-0406-4.

7. Huarte M. The emerging role of IncRNAs in cancer. Nat Med. 2015;21:125361. https://doi.org/10.1038/nm.3981.

8. Janakiraman $\mathrm{H}$, et al. The Long (IncRNA) and short (miRNA) of it: TGFbetamediated control of RNA-binding proteins and noncoding RNAs. Molecular cancer research : MCR. 2018;16:567-79. https://doi.org/10.1158/1541-7786. MCR-17-0547.

9. Dong, J. et al. HMGA2-FOXL2 Axis Regulates Metastases and Epithelial-toMesenchymal Transition of Chemoresistant Gastric Cancer. Clinical cancer research : an official journal of the American Association for Cancer Research 23, 3461-3473, doi:https://doi.org/10.1158/1078-0432.CCR-16-2180 (2017).

10. Di Cecilia S, et al. RBM5-AS1 is critical for self-renewal of Colon Cancer stemlike cells. Cancer Res. 2016;76:5615-27. https://doi.org/10.1158/0008-5472. CAN-15-1824.

11. Qiu M, et al. A novel IncRNA, LUADT1, promotes lung adenocarcinoma proliferation via the epigenetic suppression of p27. Cell Death Dis. 2015;6: e1858. https://doi.org/10.1038/cddis.2015.203.

12. Cheng $X$, et al. Circular RNA VMA21 protects against intervertebral disc degeneration through targeting miR-200c and X linked inhibitor-ofapoptosis protein. Ann Rheum Dis. 2018;77:770-9. https://doi.org/10.1136/ annrheumdis-2017-212056.

13. King VM, Borchert GM. MicroRNA expression: protein participants in MicroRNA regulation. Methods Mol Biol. 2017;1617:27-37. https://doi.org/10. 1007/978-1-4939-7046-9_2.

14. Long JM, et al. Genome-scale mRNA and small RNA transcriptomic insights into initiation of citrus apomixis. J Exp Bot. 2016;67:5743-56. https://doi.org/ 10.1093/jxb/erw338.

15. Zhang W, Le TD, Liu L, Zhou ZH, Li J. Predicting miRNA targets by integrating gene regulatory knowledge with expression profiles. PLoS One. 2016;11:e0152860. https://doi.org/10.1371/journal.pone.0152860.

16. Wang $Z$, et al. Long non-coding RNA urothelial carcinoma-associated 1 as a tumor biomarker for the diagnosis of urinary bladder cancer. Tumour biology : the journal of the International Society for Oncodevelopmental Biology and Medicine. 2017;39:1010428317709990. https://doi.org/10.1177/ 1010428317709990.

17. Wu C, Luo J. Long non-coding RNA (IncRNA) urothelial carcinoma-associated 1 (UCA1) enhances tamoxifen resistance in breast Cancer cells via inhibiting mTOR signaling pathway. Medical science monitor : international medical journal of experimental and clinical research. 2016;22:3860-7.

18. Agrawal $U$, et al. Immune signature of urothelial cancer associated with grade, recurrence, and invasion. Urol Oncol. 2016;34:418 e417-26. https:// doi.org/10.1016/j.urolonc.2016.04.013.

19. Halstead AM, et al. Bladder-cancer-associated mutations in RXRA activate peroxisome proliferator-activated receptors to drive urothelial proliferation. eLife. 2017;6. https://doi.org/10.7554/eLife.30862.

20. Li XN, Liu AH, Tang X, Ren Y. Urothelial carcinoma-associated 1 enhances tamoxifen resistance in breast cancer cells through competitively inhibiting miR-18a. Beijing da xue xue bao Yi xue ban = Journal of Peking University Health sciences. 2017:49:295-302.
21. Chen T, et al. Expression of long noncoding RNA IncRNA-n336928 is correlated with tumor stage and grade and overall survival in bladder cancer. Biochem Biophys Res Commun. 2015;468:666-70. https://doi.org/10. 1016/j.bbrc.2015.11.013.

22. Liu D, et al. LncRNA SPRY4-IT1 sponges miR-101-3p to promote proliferation and metastasis of bladder cancer cells through up-regulating EZH2. Cancer Lett. 2017;388:281-91. https://doi.org/10.1016/j.canlet.2016.12.005.

23. Su G, He Q, Wang J. Clinical values of Long non-coding RNAs in bladder Cancer: a systematic review. Front Physiol. 2018;9:652. https://doi.org/10. 3389/fphys.2018.00652.

24. Salmena L, Poliseno L, Tay Y, Kats L, Pandolfi PP. A ceRNA hypothesis: the Rosetta stone of a hidden RNA language? Cell. 2011;146:353-8. https://doi org/10.1016/j.cell.2011.07.014.

25. Gao J, et al. MicroRNA-590-5p functions as a tumor suppressor in breast cancer conferring inhibitory effects on cell migration, invasion, and epithelial-mesenchymal transition by downregulating the Wnt-beta-catenin signaling pathway. J Cell Physiol. 2018. https://doi.org/10.1002/jcp.27056.

26. Rohini M, Gokulnath M, Miranda PJ, Selvamurugan N. MiR-590-3p inhibits proliferation and promotes apoptosis by targeting activating transcription factor 3 in human breast Cancer cells. Biochimie. 2018. https://doi.org/10. 1016/j.biochi.2018.07.023.

27. Wang WT, et al. miR-590-3p is a novel microRNA which suppresses osteosarcoma progression by targeting SOX9. Biomedicine \& pharmacotherapy = Biomedecine \& pharmacotherapie. 2018;107:1763-9. https://doi.org/10.1016/j.biopha.2018.06.124.

28. Xu BB, Gu ZF, Ma M, Wang JY, Wang HN. MicroRNA-590-5p suppresses the proliferation and invasion of non-small cell lung cancer by regulating GAB1. Eur Rev Med Pharmacol Sci. 2018;22:5954-63. https://doi.org/10.26355/ eurrev_201809_15926.

29. Di Agostino S, et al. Long non-coding MIR205HG depletes Hsa-miR-590-3p leading to unrestrained proliferation in head and neck squamous cell carcinoma. Theranostics. 2018;8:1850-68. https://doi.org/10.7150/thno.22167.

30. Hay ED. An overview of epithelio-mesenchymal transformation. Acta Anat. 1995;154:8-20.

31. Nieto MA. Epithelial plasticity: a common theme in embryonic and cancer cells. Science. 2013;342:1234850. https://doi.org/10.1126/science.1234850.

32. Liu T, et al. MicroRNA-590 is an EMT-suppressive microRNA involved in the TGFbeta signaling pathway. Mol Med Rep. 2015;12:7403-11. https://doi.org/ 10.3892/mmr.2015.4374.

33. Yang B, et al. PHF14: an innate inhibitor against the progression of rena fibrosis following folic acid-induced kidney injury. Sci Rep. 2017;7:39888. https://doi.org/10.1038/srep39888.

34. Zhang $L$, et al. A novel PHD-finger protein 14/KIF4A complex overexpressed in lung cancer is involved in cell mitosis regulation and tumorigenesis. Oncotarget. 2017:8:19684-98. https://doi.org/10.18632/oncotarget.14962.

35. Akazawa T, et al. Aberrant expression of the PHF14 gene in biliary tract cancer cells. Oncol Lett. 2013;5:1849-53. https://doi.org/10.3892/ol.2013.1278.
Ready to submit your research? Choose BMC and benefit from:
- fast, convenient online submission
- thorough peer review by experienced researchers in your field
- rapid publication on acceptance
- support for research data, including large and complex data types
- gold Open Access which fosters wider collaboration and increased citations
- maximum visibility for your research: over $100 \mathrm{M}$ website views per year
At BMC, research is always in progress.
Learn more biomedcentral.com/submissions 